\title{
Diseño de un modelo de transferencia Universidad-Empresa, para Ia I+D generado por grupos de investigación de la Universidad de Pamplona
}

FECHA DE RECEPCIÓN: 8 de marzo FECHA DE APROBACIÓN: abril 20 Pp. 106-119

\section{Javier Mauricio García Mogollón* Carlos Andrés Gualdrón Guerrero** Rafael Bolívar León ${ }^{* * *}$}

Design of a 'University-

enterprise" transfer mode/ for the $1+\square$ generated by research groups from the University of Pamplona

Création d'un modèle de transfert "Lniversité-Entreprise » pour la RED réalisé par différents gruupes de recherche de I'Université de Pampelune.

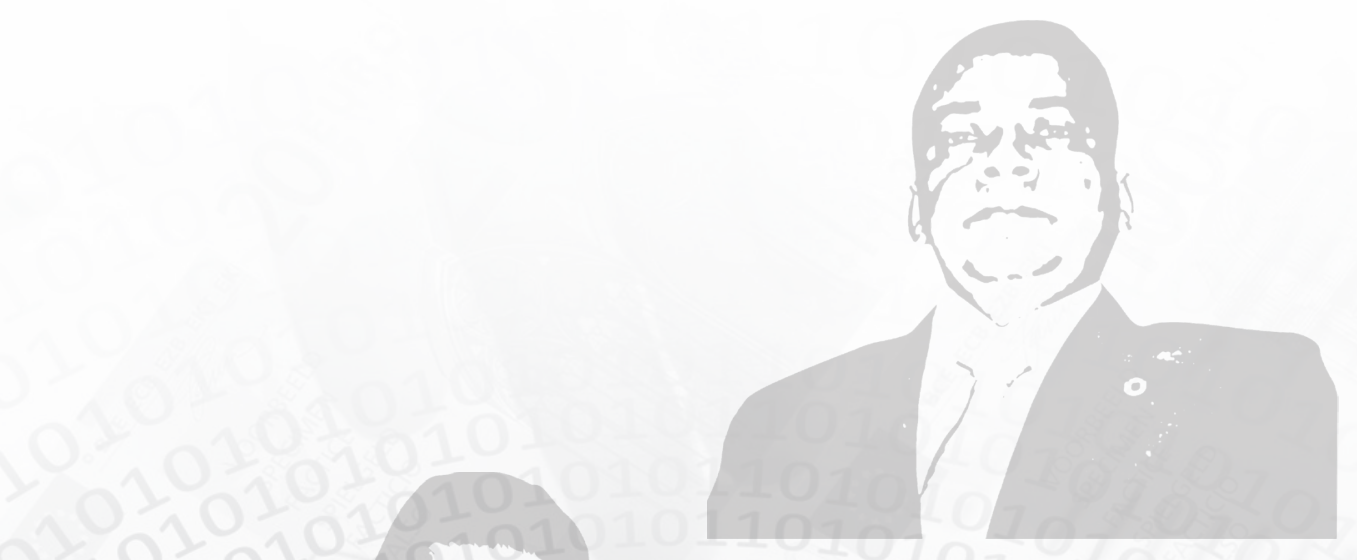

Desenho de um modela de transferência 'Universidade-Empresa' para a $1+D$ gerado por grupos de investigaçãa da Universidade de Pamplana
"Mágister en Ciencias de la Administración de la Universidad de los Andes-Mérida Venezuela; Administrador comercial, Universidad de Pamplona; Docente de planta de la Universidad de Pamplona.

**Doctor en Economía, Universidad de Alicante-España; Economista, Universidad Santo Tomás de Bucaramangas, Docente de planta Universidad de Pamplona.

*** Doctor en procesos metalúrgicos y reciclaje de metales-RWTH-Aachen-Alemania; Ingeniero metalúrgico, Universidad Industrial de Santander; Docente de planta Universidad de Pamplona. 


\section{RESUMEN}

Este trabajo tiene como objetivo presentar un modelo de transferencia 'UniversidadEmpresa' para la Universidad de Pamplona en Norte de Santander. Bajo esta premisa, se busca contribuir más allá de los planteamientos teóricos de la transferencia, trasladando los resultados de manera sistémica a un modelo. Este estudio de carácter cualitativo muestra la extracción de la realidad en la percepción de los investigadores con respecto a la estructura de la transferencia de investigación. Se definieron categorías relacionadas con la cultura de investigación, política en la investigación y emprendimiento. Estas permitieron a través de un proceso de codificación abierta y selectiva, refinar un modelo que pretende integrar procesos aislados con respecto a la gestión de la investigación de la misma universidad.

\section{ABSTRACT}

This research paper aims at presenting a relational- transfer University-Company model for Pamplona University, located in Northern Santander. It tries to go beyond the theoretical bases of transfer, designing a model out of the systemic results found. This qualitative study shows the segregation of the researchers' perception from the objective reality when experiencing the transfer structure of the process of research. Categories such as Research Culture, Research Policy and Entrepreneurship have been defined. This allows through an open and selective process to improve a model which integrates isolated processes with regard to the research management at Pamplona University.

\section{RESUMÉÉ}

Cette étude a pour objectif de présenter un modèle de transfert "Université-Entreprise " pour l'université de Pampelune, Santander du Nord. L'objectif de l'étude entend contribuer au transfert systémique des résultats théoriques à un modèle. Cette étude à caractère qualitatif présente l'extraction de la réalité dans la perception des chercheurs quant à la structure du transfert de recherche. Les catégories définies sont liées à la culture de recherche sur les politiques et l'esprit d'entreprise mis en place grâce à un processus de codage ouvert et sélectif visant à améliorer un modèle d'intégration de la gestion de la recherche universitaire.

\section{RESUMO}

Oobjetivo deste trabalho é apresentar um modelo de transferência 'Universidade-Empresa' para a Universidade de Pamplona em Norte de Santander. Segundo esta premissa, procurase contribuir para além das concepções teóricas da transferência, trasladando os resultados de maneira sistémica para um modelo. Este estudo de tipo qualitativo mostra a extração da realidade na percepção dos pesquisadores com respeito à estrutura da transferência de investigação. Concretizaram-se categorias relacionadas à Cultura de Investigação, Política na Investigação e Empreendimento. Estas categorias permitiram através de um processo de codificação aberta e seletiva refinar um modelo que tem como objetivo integrar processos isolados com respeito à gestão da investigação da própria universidade.

\section{Palabras claves}

Estrategia social

Transferencia de conocimiento Modelos organizacionales Sistemas organizacionales Generación de impacto

\section{Key words}

Social strategy

Knowledge transference Organizational models Organizational systems Impact generation

\section{Mots clefs}

Stratégie sociale Transfert de connaissances Modèles organisationnels Systèmes organisationnels

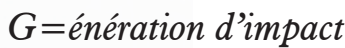

Palavras-chave

Estratégia social Transferência de conhecimento Modelos organizativos Sistemas organizativos Geração de impacto 


\section{INTRODUCCIÓN}

A ctualmente se reconoce la existencia a nivel mundial de estructuras que permiten el vínculo entre diferentes actores: Universidad, Empresa y Estado. En Colombia se presenta el caso donde las universidades buscan que los proyectos formulados y ejecutados transfieran conocimiento y generen impacto en todos los ámbitos, utilizando diversidad de sistemas organizacionales como: centros o institutos de investigación, polos de innovación, empresas de base tecnológica, entre otras.

Una de las misiones actuales de la universidad es lograr mayor conexión entre la formación y el desarrollo profesional de sus estudiantes y docentes. También hace parte de la gestión universitaria la investigación que históricamente está relacionada entre el sistema científicotecnológico y la sociedad en su conjunto; está concebida como estrategia social para la superación de la pobreza y el atraso social. Además, es importante mencionar otro proceso misional que hace referencia a la proyección social, esta última surgida de la responsabilidad social empresarial, para la cual la universidad se apropia del concepto y le da un enfoque de extensión y proyección social como complemento intencionado en la formación estudiantil y, por su naturaleza, la creación y transferencia del conocimiento a través de la investigación. Estos tres procesos misionales de formación, proyección social e investigación, están estrechamente relacionados con la sociedad que exige a la universidad la transferencia del conocimiento para mejorar las condiciones dignas de la vida humana en una comunidad.

Dentro de ese condicionamiento la universidad, en su evolución como organización que genera y transmite conocimiento, ha tenido que desarrollar en su interior modelos

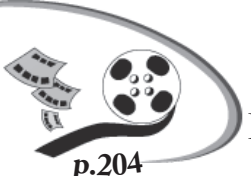

p.204 organizacionales que le permitan dar respuesta a las existencias de la sociedad. Esta afirmación la justifican López, Mejía y

Schmal (2006): "hasta hace poco, la sociedad le asignó formalmente a las universidades y a los centros de investigación la misión casi exclusiva de producción de conocimiento".
La Universidad de Pamplona, en su proceso misional de investigación, que de alguna manera se ve reflejado en este proyecto, permite reconocer la necesidad de transferir conocimiento como modelo ideal no solo para resultados de investigación aplicada; además, genera espacios de integración social que permiten la generación de empleo, el mejoramiento de calidad de vida y otros factores que exige la sociedad en la actualidad.

Se ha comenzado a buscar ese ideal que tiene la universidad frente a la sociedad. Es el caso explícito de resultados de trabajos de investigación que originaron este proyecto, como lo evidencian en su trabajo "El ayer y el hoy de la integración Universidad-Empresa" García y Durán (2011), en el que se describió la evolución de los vínculos y transferencia desde el nacimiento de la universidad hasta analizar las estructuras modernas que permiten vincular y transferir conocimiento desde la universidad a la empresa. Otra aproximación que profundiza el tema de la transferencia desde el enfoque interno en las organizaciones que generan conocimiento lo esboza García (2012), a través de estrategias de mercadeo interno que mejoran las condiciones de la problemática organizacional de los institutos de investigación de la Universidad de Pamplona. Estos trabajos han permitido aclarar que los asuntos de cambios organizacionales en la gestión de investigación, deben iniciar internamente con procesos de mejora continua para luego dar respuesta de manera eficiente a las exigencias de la sociedad.

En cierta manera se pretendió dar respuesta a los propósitos de esta investigación. En primera instancia se buscó reconocer la existencia teórica de modelos de transferencia; seguidamente se trató de traducir la percepción de un modelo de transferencia por parte de los investigadores de la universidad, y como último propósito, se definió un modelo que surge de la realidad humana y organizacional que puede dar respuesta a esas exigencias que tanto le hacen falta a la región del nororiente colombiano. 
Para tener una visión general se estructuró este documento de la siguiente manera. En la primera referencia, el ítem de Revisión Literaria, amplía la temática con la exploración teórica, conceptual y de antecedentes con diversas posturas, desde lo empresarial, el Estado y lo universitario. A continuación se presenta una explicación metodológica del tipo de investigación cualitativa que se aplicó con herramientas que lograron capturar información y permitieron definir el modelo. En la tercera parte, se presentan los resultados de investigación, mostrando de manera gráfica el modelo y su respectiva explicación. Finalmente, se plasman las conclusiones.

\section{REVISIÓN LITERARIA}

$\mathbf{L}$ a fundamentación teórica de este trabajo, resalta la revisión literaria, la cual se muestra en tres contextos. Inicialmente, se presenta una posición conceptual donde se tratan modelos que han desarrollado la teoría de la transferencia. Al final se definieron tres posturas que surgen de las experiencias. La primera, con respecto a la identificación de tipo de empresa a trasferir conocimiento, la segunda, plasma la intención del Estado en Colombia con respecto al compromiso de apoyar la trasferencia de conocimiento y la tercera, define la responsabilidad de la universidad.

\subsection{Modelos de transferencia}

En este apartado se pretende hacer una exploración del término transferencia; posteriormente se abordan los modelos básicos más relevantes y finalmente, se profundiza con la postura que presenta la empresa, el Estado y la universidad con respecto al proceso de transferencia.

Para fundamentar el modelo, necesariamente se ilustra el significado de 'Transferencia de investigación aplicada' desde su contexto, como lo plantean López, Mejía y Schmal (2006), quienes lo definen como un proceso mediante el cual el sector privado obtiene el acceso a los avances tecnológicos desarrollados por los científicos, el cual es un nexo entre la universidad y las empresas para la generación de desarrollo científico-técnico y económico. La transferencia conlleva un convenio, un acuerdo, presupone un pago y por tanto, la comercialización del conocimiento es un elemento inherente a este proceso.

Ampliando la anterior cita, el concepto de transferencia tiene diversas actividades que involucran a la universidad con la empresa. Algunos autores definen procesos de transferencia a partir de sus experiencias, como lo plantea Morales (2008), quien identifica los modelos de transferencia definiendo procesos de investigación contratada, macroproyectos, consultoría, enseñanza externa, presentación de servicios especializados, entre otros. Ortín, Salas, Trujillo y Vendrell (2008) definen los modelos de transferencia en el emprendimiento que surge de los procesos de infestación a través de las Spin Off (emprendimiento surgido de la investigación). Otra noción del concepto de transferencia la relacionan Luna y Solleiro (2007), quienes presentan los procesos de gestión de investigación en relación con patentes, licencias, alianzas, regalías, derechos de autor, venta de tecnología. En otra postura planteada por Göktepe (2005) se identifican factores como el licenciamiento, generado por las universidades, la creación de empresas, Start-up (consultoría a empresas), las movilidades, entre otras actividades relacionadas con el tema. Finalmente, se plantea el concepto de transferencia por Calderón (2005), quien apunta a los vínculos con las empresas formales, la captación de recursos a través de las fundaciones y el emprendimiento surgido de las Spin-off. Esta diversidad conceptual hace que el tema en la actualidad sea amplio con diversos criterios, conceptos, experiencias, que de alguna manera logran la existencia de un fundamento cierto para la transferencia de una gran diversidad de procesos que se mencionan por los autores y que hacen necesaria la teorización de los modelos que permitan dinamizar los resultados de la investigación aplicada.

A continuación se presentan los modelos básicos de transferencia que dan un soporte teórico al diseño del modelo. Inicialmente, se referenciará el Modelo Lineal, luego el Triángulo de Sábato y seguidamente, el planteamiento de La Triple Hélice. 
El Modelo Lineal se plantea como un modelo de innovación que se caracteriza por definir la trasferencia, partiendo de las necesidades del mercado, acompañada de la investigación básica, para luego llevarla a la investigación aplicada e iniciar el proceso de transferencia de los resultados de investigación (figura 1).

\section{Figura 1. Modelo Lineal}

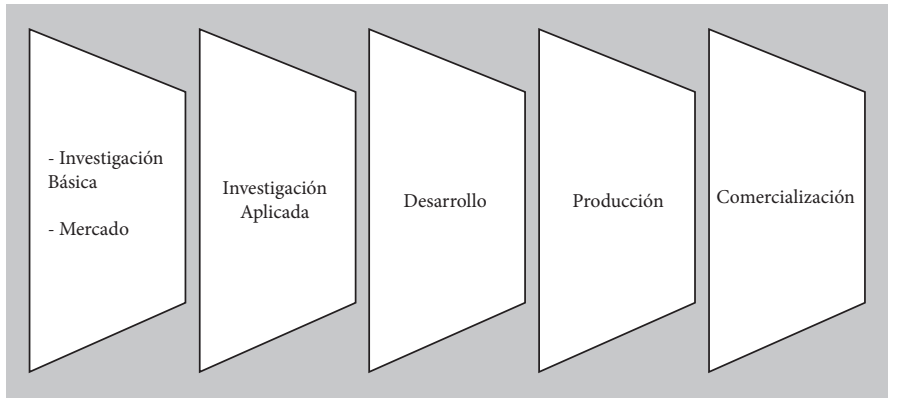

Fuente. Smith (1995).

Este enfoque considera que para ingresar al mercado nuevos productos, o para modificar los procesos de fabricación, se plantea una serie de etapas que se inician con la investigación científica; por ello, este enfoque se denomina Lineal, ya que se considera que a partir de las actividades de $\mathrm{I}+\mathrm{D}$, se ha de llegar necesariamente a la incorporación al mercado de nuevos productos o procesos. Este modelo, en su funcionalidad, no refleja un proceso de traslado de resultados de investigación a las empresas, lo cual deja a un lado mecanismos formales que logren el objetivo del vínculo en la universidad y la empresa; algunos autores la denominan como un sistema que nace de la investigación básica. El caso contrario es evidenciado en el Triángulo de Sábato que a continuación se profundiza.

Dentro del planteamiento de transferencia y su evolución se presenta la necesidad de pasar de procesos internos a dinamizar el modelo con factores

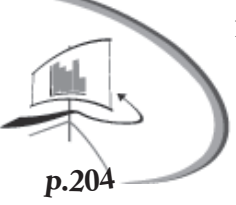

como el gobierno y su interacción con la empresa y la universidad. Según Solleiro (2008), el Triángulo de Sábato (figura 2),

es quizás el modelo más elemental y universalmente aceptado en la política científica-tecnológica; se basa en la idea de que uno de los motores del desarrollo radica en los vínculos entre el gobierno, la estructura productiva y las instituciones.
Figura 2. Triángulo de Sábato

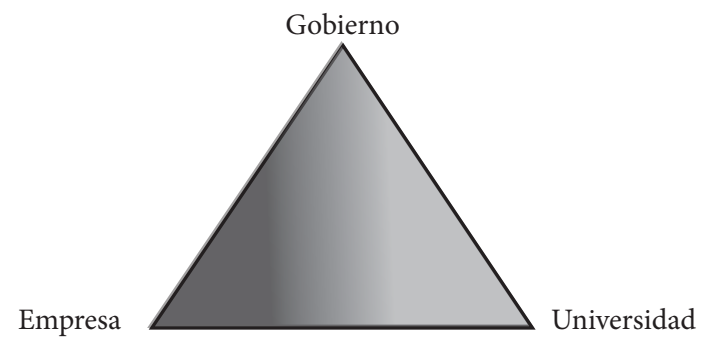

Fuente. Solleiro (2008).

De esta manera, la figura presenta un modelo orientador de las estrategias de desarrollo. Está representado mediante un triángulo de interacciones entre los vértices correspondientes al gobierno, a la estructura productiva y a la infraestructura científico-técnica. Las interrelaciones entre los vértices permiten crear un flujo de demandas y ofertas que conduce a la generación y utilización de conocimientos estratégicos y socialmente útiles.

Dentro de las proposiciones que se manifiestan a través del tiempo surge otro modelo denominado "La Triple Hélice", propuesto por Leydesdorff y Etzkowitz (1998). Ellos tratan el tema de un modelo de innovación y transferencia. Su análisis es inherente en este tema puesto que explica la correlación de las fuerzas que apalancan la generación del conocimiento a transferir. En este modelo, sus autores han presentado una evolución donde definen la complejidad que surge de la inserción de la ciencia y la tecnología en el sector productivo. El Estado crea instituciones públicas de investigación, las universidades crean empresas y estas a su vez, crean unidades de investigación y desarrollo (Etzkowitz y Leydesdorff, 2000).

El modelo denominado "La Triple Hélice" (figura 3), en la actualidad tiene vigencia a nivel internacional, donde uno de sus autores -el profesor Leydesdorff, asociado a la University of Amsterdam- demuestra a la comunidad científica que los vínculos entre la universidad, la empresa y el estado necesariamente están atados a la economía global, teniendo como reto construir "el estado emprendedor" (Triplehelix, 2013). En esta postu-ra que pasa de lo epistemológico a la praxis,

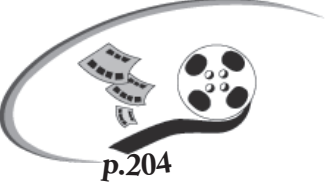
se expone una visión contemporánea de la transferencia; se manifiesta la necesidad del emprendimiento germinado dentro de la universidad con el apoyo del estado para dar origen a las empresas innovadoras. 
Figura 3. Representación esquemática del concepto de "La Triple Hélice" en el sistema de ciencia-tecnología-empresa

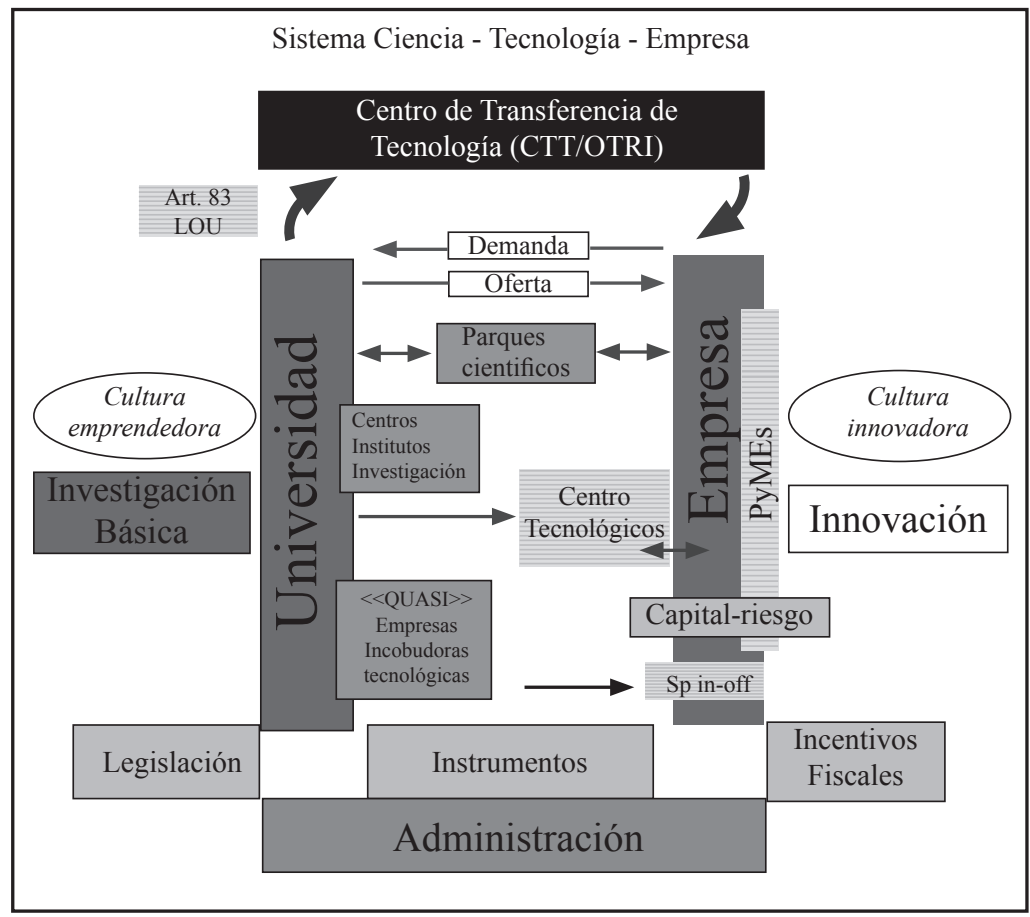

Fuente. COTEC (2003).

Se exponen las estructuras de intermediación que escenifican los modelos de transferencia en España, (figura 3): parques científicos, incubadoras tecnológicas, centro tecnológico, entra otras. La empresa, la universidad y la administración (Estado), son los actores determinantes para que la cultura innovadora dé respuesta a una oferta y demanda de productos y servicios donde las estructuras de intermediación puedan apoyar y generar una posible comercialización con soporte legal y de incentivos fiscales por parte del Estado.

Para el caso de Latinoamérica, se caracteriza el modelo dentro de una serie de actividades que vinculan la universidad y la empresa (figura 4).

En este modelo la universidad tiene como misión dos actividades base para la transferencia: en primera instancia la de vinculación, y en segunda, las actividades de propiedad intelectual. La empresa en este modelo está intervenida por una serie de actividades que permiten establecer mercados, incubar empresas, hacer consultoría, entre otros.
Para el caso de Colombia, la existencia de modelos de transferencia en algunas universidades es un hecho evidenciado desde lo educativo a nivel de posgrado con la oferta de especializaciones en el tema de gerencia de tecnología, centros de innovación, consultoría y empresarismo, centros de investigación y desarrollo, vicerrectorías de investigación de las universidades, grupos de investigación, semilleros de investigación, entre otras actividades.

En algunos casos, dentro de las mismas Instituciones de Educación Superior (IES), estas actividades están desarticuladas por la falta de conocimiento y políticas de un modelo que permitan la transferencia de resultados de investigación universitaria. El anterior planteamiento lo profundiza Tognato (2005), quien expresa que la comercialización de la tecnología es una oportunidad que las universidades colombianas aún no han desarrollado sistemáticamente. Para aprovechar esta oportunidad es necesario que las universidades se encaminen en un proceso de ajuste y de aprendizaje institucional, llenando así las brechas que actualmente impiden explotar eficaz y eficientemente la propiedad intelectual generada a través de las actividades de investigación. 
Figura 4. Modelo de transferencia de tecnología latinoamericano

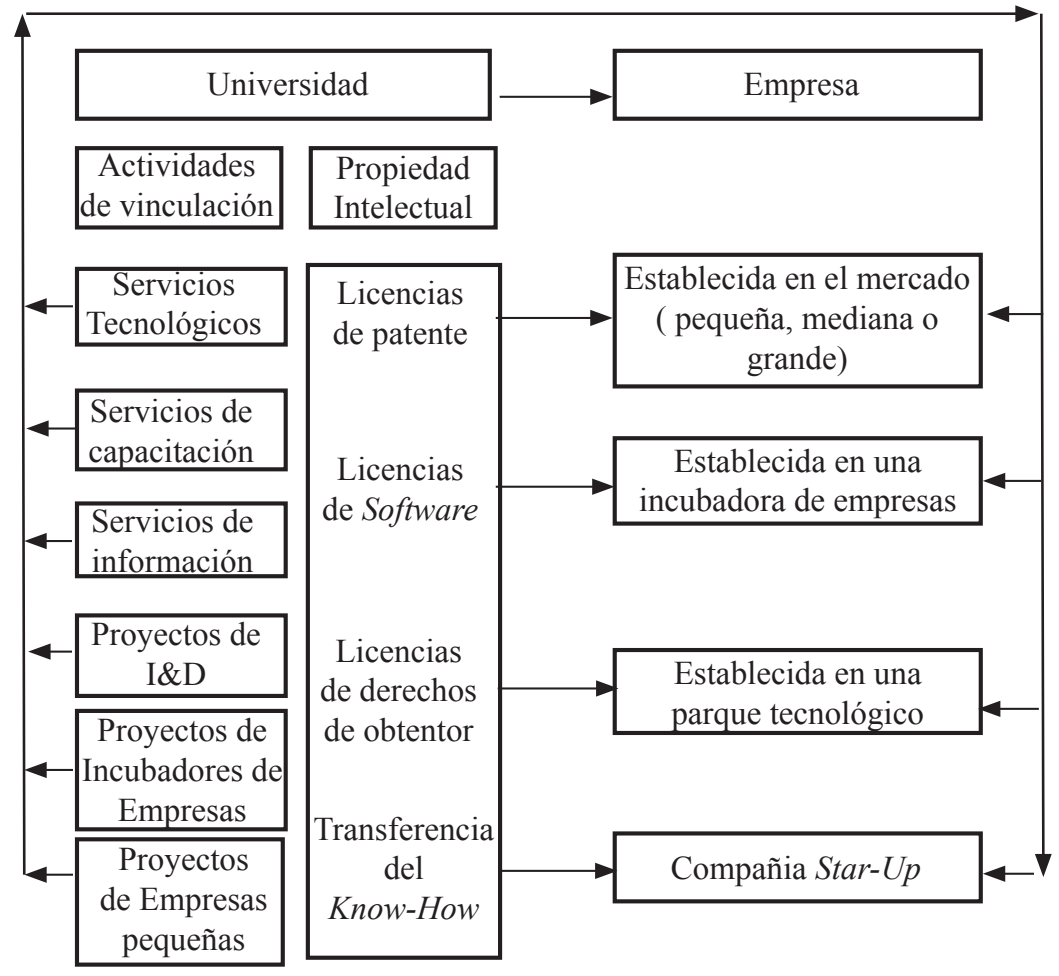

Fuente. Solleiro y Castañon (2008).

En este aparte se puede concluir parcialmente que los modelos planteados permitieron categorizar lo metodológico, logrando estructurar y definir procesos que orientan la transmisión, la venta, la incorporación de conocimientos tecnológicos y científicos necesarios para la existencia de mecanismos que conduzcan a generar un impacto en los grupos de interés y así, poder comprender la naturaleza del sistema de vínculo y transferencia local. A continuación se presentan las posturas de los actores de la transferencia.

\subsubsection{Postura empresarial de transferencia}

La existencia de diferentes tipos de empresas puede definir cuál sería el impacto que tendría la intervención de la universidad. Jasso (2004) presenta un análisis de la trayectoria y el ciclo de vida de estas, que son determinantes para definir un posible socio en la relación Universidad-Empresas. Otros autores las segmentan desde diversas variables (tabla 1).

\section{Tabla 1. Tipos de empresas para la transferencia}

\begin{tabular}{|c|c|}
\hline Tipo 4 - Excelentes & Tipo 2 - Confiables \\
\hline $\begin{array}{l}\text { Son los grandes jugadores. Les } \\
\text { interesa proyectos de I+D+i } \\
\text { (para lo cual generalmente } \\
\text { tienen sus propios equipos) } \\
\text { y por tanto, ya no buscan a la } \\
\text { universidad, puesto que hacen } \\
\text { inversiones directas y luego las } \\
\text { deducen por beneficios sobre } \\
\text { inversión directa e innovación. }\end{array}$ & $\begin{array}{l}\text { Tienen una estructura básica } \\
\text { definida y formalizada que } \\
\text { requiere diseño de producto, } \\
\text { mejoramiento continuo de pro- } \\
\text { cesos, cambio de tecnología, } \\
\text { incremento del volumen de } \\
\text { facturación. Son empresas con } \\
\text { más de tres años en el mercado. }\end{array}$ \\
\hline Tipo 3 - Competitivas & Tipo 1 - Incipientes \\
\hline $\begin{array}{l}\text { Son las empresas que tienen } \\
\text { posicionamiento; asisten a las } \\
\text { ruedas de negocios porque ya } \\
\text { hablan de innovación. Ellas } \\
\text { piensan en normas ISO, inte- } \\
\text { ligencia de mercados y planes } \\
\text { de exportación. }\end{array}$ & $\begin{array}{l}\text { Artesanales, empíricas; toda- } \\
\text { vía no se atienden con trasfe- } \\
\text { rencia de tecnología. Recla- } \\
\text { man lo básico: organización } \\
\text { administrativa, financiera, con- } \\
\text { table, mercadeo y talento hu- } \\
\text { mano. Pueden ser atendidas } \\
\text { con estudiantes de últimos } \\
\text { semestres, vinculados a exten- } \\
\text { sión de las universidades. }\end{array}$ \\
\hline
\end{tabular}

Fuente. Benavente (2004). 
Como se visualizó anteriormente (tabla 1), Benavente (2994) segmentó el sector empresarial en cuatro tipos. Se puede inferir dentro del planteamiento anterior que la universidad que pretenda transferir ciencia y tecnología debería considerar las empresas tipo 3 y tipo 4; son estas las que presentan la pertinencia para desarrollar proyectos en conjunto Universidad-Empresa-Estado.

Es así como se reseña la posibilidad de transferencia al sector productivo de los resultados de los proyectos de investigación aplicados de las universidades, con el apoyo de Estado.

\subsubsection{Postura del Estado en la transferencia}

Por otra parte, el enfoque del factor Estado ha "descentralizado" la entrega de recursos para proyectos de Ciencia y Tecnología a través de las Gobernaciones y diversos programas de gobierno que buscan canalizar recursos para fortalecer las PyMEs a través del desarrollo de proyectos de $\mathrm{I}+\mathrm{D}+\mathrm{i}$, entre otros. En este contexto, las universidades deben buscar financiamiento para trasladar la investigación aplicada al sector empresarial, donde estén presentes las reglas formales que generen confianza entre las partes y permitan que la universidad presente un mejoramiento en los procesos presentes en las organizaciones competitivas y de excelencia.

En Colombia, se ha evidenciado una evolución normativa desde la capacidad y posibilidades que presenta el Estado colombiano frente a la necesidad de transferir conocimiento. La Ley 1286 de 2009, en su artículo 18 "Las Actividades del Sistema Nacional de Ciencia, Tecnología e Innovación", en su parágrafo 3, plantea: "Velar por la generación, transferencia, adaptación y mejora del conocimiento científico, desarrollo tecnológico e innovación en la producción de bienes y servicios para los mercados regionales, nacionales e internacionales".

Los procesos de transferencia se caracterizan por tener el apoyo del factor Estado en Colombia; aunque las generadoras de conocimiento implementen y desarrollen mecanismos e instrumentos que les permitan presentar políticas dentro de las mismas universidades para que se dinamice el espacio, como el que se presenta desde el 2007 por iniciativa de Ministerio de Educación Nacional y Colciencias, a través del Comité Universidad, Empresa y Estado (UEE).
Se pretende facilitar sinergia de conocimiento y voluntades entre los actores: sector empresarial, las universidades y el gobierno que está representado en cada departamento por las gobernaciones. Esta relación ha permitido dinamizar los vínculos y la transferencia Universidad-Empresas en los departamentos de Santander, Norte de Santander, Valle del Cauca, Antioquia, Eje cafetero, Atlántico, Bogotá, entre otros departamentos. La existencia de agendas en los procesos de transferencia de tecnología es un hecho en Colombia. Dentro de algunas aportaciones esta se define:

Cuando hablamos de transferencia de tecnología, nos referimos a todos aquellos procesos necesarios para que el sector productivo pueda acceder a los nuevos desarrollos tecnológicos que mejoren y sustenten su actividad. Dada la alta especialización de esta tecnología y la necesidad ...lo que exige que las universidades sean las instituciones que con-tribuyan de manera significativa a favorecer esta transferencia, pues cuentan con el recurso humano preparado para generar, adaptar y transferir la tecnología que requiere el desarrollo nacional (Calderón, 2005).

Es evidente la importancia del talento humano para que se logre estimular un desarrollo tecnológico en un país que posibilite la autonomía relativa de las actividades productivas y de los servicios ineluctables para alcanzar y sostener el desarrollo. Todo esto a través del fortalecimiento de la capacidad de innovar, importar, modificar y divulgar tecnologías orientadas a la satisfacción de las necesidades humanas que, a su vez, favorezcan el desarrollo científico, donde la importancia de la formalización de las relaciones entre Universidad-Empresa, la existencia de contratos, el reconocimiento de los límites de la comercialización, y la diseminación de tecnología al sector productivo sean una realidad (Teruya, 2010).

\subsubsection{Postura de la universidad en la transferencia}

Vega, Fernández y Huanca (2007) plantean en las conclusiones de su proyecto, la poca consolidación de la investigación como actividad universitaria, la existencia de reticencias internas en la comunidad docente para la adopción de prácticas empresariales y la débil demanda de conocimiento tecnológico y baja capacidad de absorción del sector productivo. 
Dentro del contexto colombiano, Arias y Aristizabal (2011), en su trabajo, concluyen que el concepto de innovación bajo el cual opera una institución de educación superior no puede estar estrictamente circunscrito al contexto empresarial y ligado exclusivamente a valores como la competitividad y la productividad. Otra de sus deducciones es que los mecanismos de transferencia más utilizados en la Instituciones de Educación Superior (IES) son la divulgación y la contratación pública para la realización de proyectos sociales.

Llegando a este punto, se puede deducir la existencia de modelos de transferencia de investigación aplicada. Esta última es la responsable, en cierta medida, de que los resultados de investigación tengan un destino que evidentemente no deja de ser un sistema organizacional fragmentado, que para algunos casos no está plenamente identificado en las universidades. Una referencia que amplía la naturaleza de la transferencia de resultados en organizaciones universitarias señala que:

Una anatomía más apropiada incluiría más integración vertical; menos colaboraciones pero más largas y estrechas; que las universidades privilegien el compartir más que el patentar los descubrimientos; más investigación interdisciplinaria; más financiamiento privado y federal para la investigación trasnacional, que conecta a las ciencias básicas y aplicadas. Así, la ciencia puede ser un negocio (Gary, 2007, p. 37-43).

Desde una postura holística, los fenómenos que surgen de la realidad organizacional de la Universidad de Pamplona, son reducibles a sistemas interdependientes que crean un sistema como un todo integrado y global. Este planteamiento ha permitido extraer de la realidad un modelo de transferencia.

\section{METODOLOGÍA}

E ste proyecto presenta una posición relativista en la cual se pretende dar una validez subjetiva en el contexto en los sistemas de transferencia de investigación universitaria, específicamente de la Universidad de Pamplona y de su proceso misional, denominado Gestión de Investigación. Como se evidencia en la investigación, se asigna la utilización de un método de investigación cualitativo. En su desarrollo, este generó una codificación abierta para el proceso analítico de información suministrada por los entrevistados, que a su vez permitió conceptualizar y categorizar los resultados para luego pasar a una codificación selectiva. Los entrevistados que participaron en el proyecto fueron investigadores reconocidos de la Universidad de Pamplona, Norte de Santander. Se tuvo en cuenta la clasificación disciplinar de los grupos de investigación como: ciencias naturales, ciencias sociales, ciencias formales y humanidades.

Se lograron identificar las siguientes categorías: reconocimiento de los procesos de administración y gestión para la investigación en la Universidad de Pamplona; percepción de la cultura de investigación; política en la investigación y emprendimiento. Cada una de ellas con diversidad de subcategorías. Para la estructuración de las categorías se tiene como referente a Rincón de Parra (2004), cuyo trabajo evalúa la transferencia del conocimiento en la relación de cooperación Universidad-Empresa. También se hace alusión del tema en el trabajo realizado por Correo y Arango (2008), quienes plantean el diseño de un modelo de gestión de conocimiento; por último, se tuvo en cuenta el Plan Estratégico 2013 de Investigación de la Vicerrectoría de Investigaciones de la Universidad de Pamplona, que presenta un modelo interno.

Posteriormente, se dio inicio al proceso de codificación de la información recolectada en el trabajo de campo con la opción de entrevista no estructura. Luego, se buscó reducir la información en fragmentos que fueron etiquetados $\mathrm{y}$ esto, permitió seleccionar apartados de texto para generar la codificación selectiva. En este proceso se logró tamizar y definir, de manera gráfica, la realidad prospectiva de modelo de transferencia y los vínculos internos y externos que intervienen en el proceso. 


\section{RESULTADOS}

Se representan los resultados generados por el descuObrimiento de categorías bajo términos explicativos más abstractos, agrupados delasiguientemanera:emprendimiento, vínculos y transferencia, gestión tecnológica, política interna de ciencia y tecnología. Estas categorías son la representación del fenómeno originado por los entre-vistados, soportadas por referencias y por la teoría (figura 5).

Dentro de esta investigación cualitativa, para simplificar el modelo, inicialmente se tuvieron en cuenta los resultados de la entrevista abierta. Este proceso permitió reducir grandes cantidades de datos a partes pequeñas y manejables, las cuales se presentan en 17 horas de grabación trasladadas a texto y procesadas para el manejo de la información cualitativa en Atlas.ti.

Concluido el proceso de codificación abierta se empezó la codificación selectiva. Esta permitió integrar y refinar la teoría consistente en las categorías mencionadas con el fin de construir el modelo simplificado para su respectivo análisis.

Figura 5. Modelo de transferencia de la Universidad de Pamplona

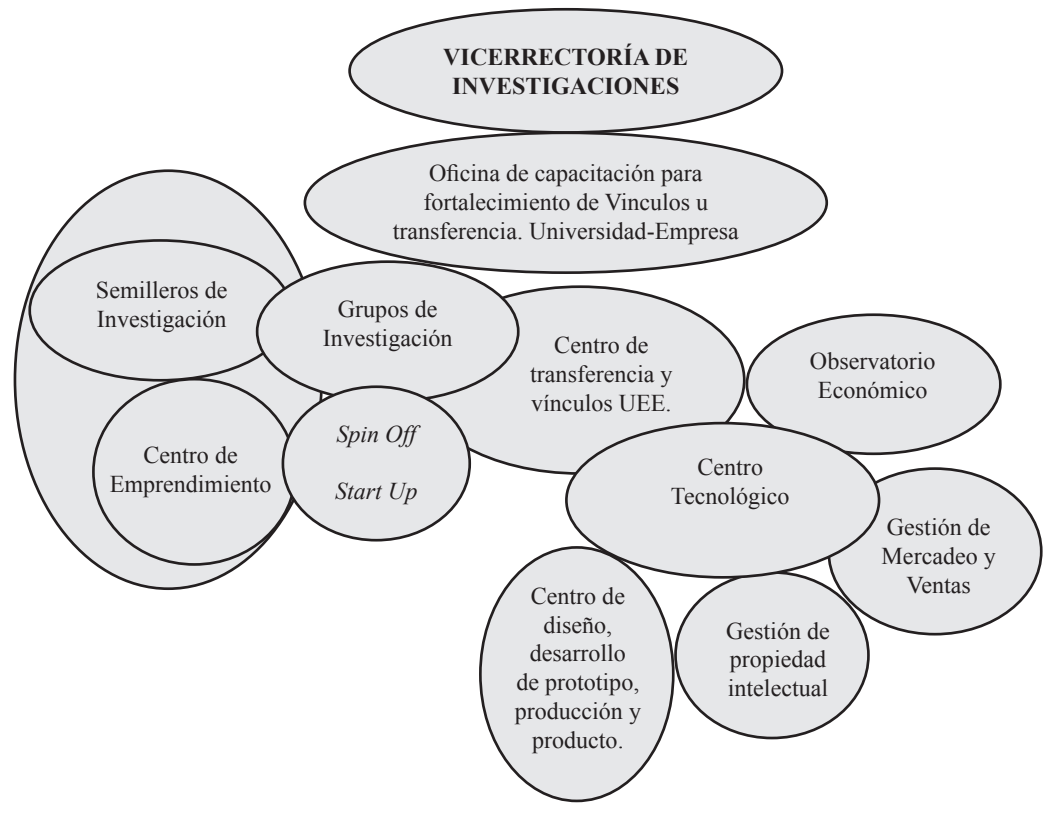

Fuente. Elaboración propia.

Para lograr definir el modelo planteado, se pretendió diseñar un esquema "Modelo de transferencia" (figura 5) originado de la realidad en el entorno, buscando una aproximación real.

Inicialmente, dentro del diseño de este modelo, se identificó que la responsable del proceso es la Vicerrectoría de Investigación. Esta dependencia tendrá como núcleo del proceso de transferencia el Centro de Transferencia y Vínculos UEE (Fundación). La finalidad de dicho centro es enlazar las actividades de investigación generadas por los grupos de investigación de la Universidad de Pamplona. Así mismo, contaría con el apoyo del Centro Tecnológico (Fundación) que, junto al Centro de Emprendimiento, vincularía las ideas de negocio canalizado a través de las Spin Off y Start Up. Estas ideas de negocio y negocios emergentes se trasladarán al Centro Tecnológico temporalmente hasta que maduren y puedan mantenerse inicialmente en el mercado regional, luego en el nacional y posteriormente en el internacional. 
Continuando con la descripción del modelo, se identificó una categoría denominada "Cultura en la investigación", la cual admitió definir una estructura que permitiría dar a conocer el funcionamiento en la gestión del modelo de transferencia y además, sensibilizar y capacitar a todos los actores interesados en iniciar procesos de transferencia: comunidad estudiantil (semilleros), la academia y comunidad científica; esto se lograría con el apoyo de la oficina de Capacitación para el Fortalecimiento de Vínculos y Transferencia.

Dentro del sistema proyectado se logró plantear el Centro Tecnológico, que tendrá como misión diseñar, patentar, desarrollar y mercadear prototipo-producto y prestar a los clientes un soporte o garantía de servicio o producto transferido. En sus procesos tendría como responsabilidad de identificación de la oferta tecnológica que demande el sector productivo la gestión de recursos, la gestión de mercadeo y su venta como producto y/o servicio; diseñar, desarrollar productos que permitan reducir la dependencia tecnológica foránea y generar un impacto en el sector productivo del departamento.

En el Observatorio Económico, adscrito a la Facultad de Ciencias Económicas y Empresariales, sus investigadores generan un sistema que precisa formular y evaluar proyectos de actuación de política económica de la región. Esto indica que las propuestas generadas por la universidad y/o el sector empresarial pueden previamente tener un panorama en ámbitos empresariales, financieros, académicos, públicos y estadísticos de la región binacional del departamento de Norte de Santander.

Estas estructuras posibilitan que se valoren los resultados de investigación generados por la universidad, obteniendo la inserción de conocimiento al sector empresarial y teniendo un alto grado de credibilidad por parte del sector productivo, presentándole una estructura que beneficiaría mutuamente a los actores donde el riesgo es reducido.

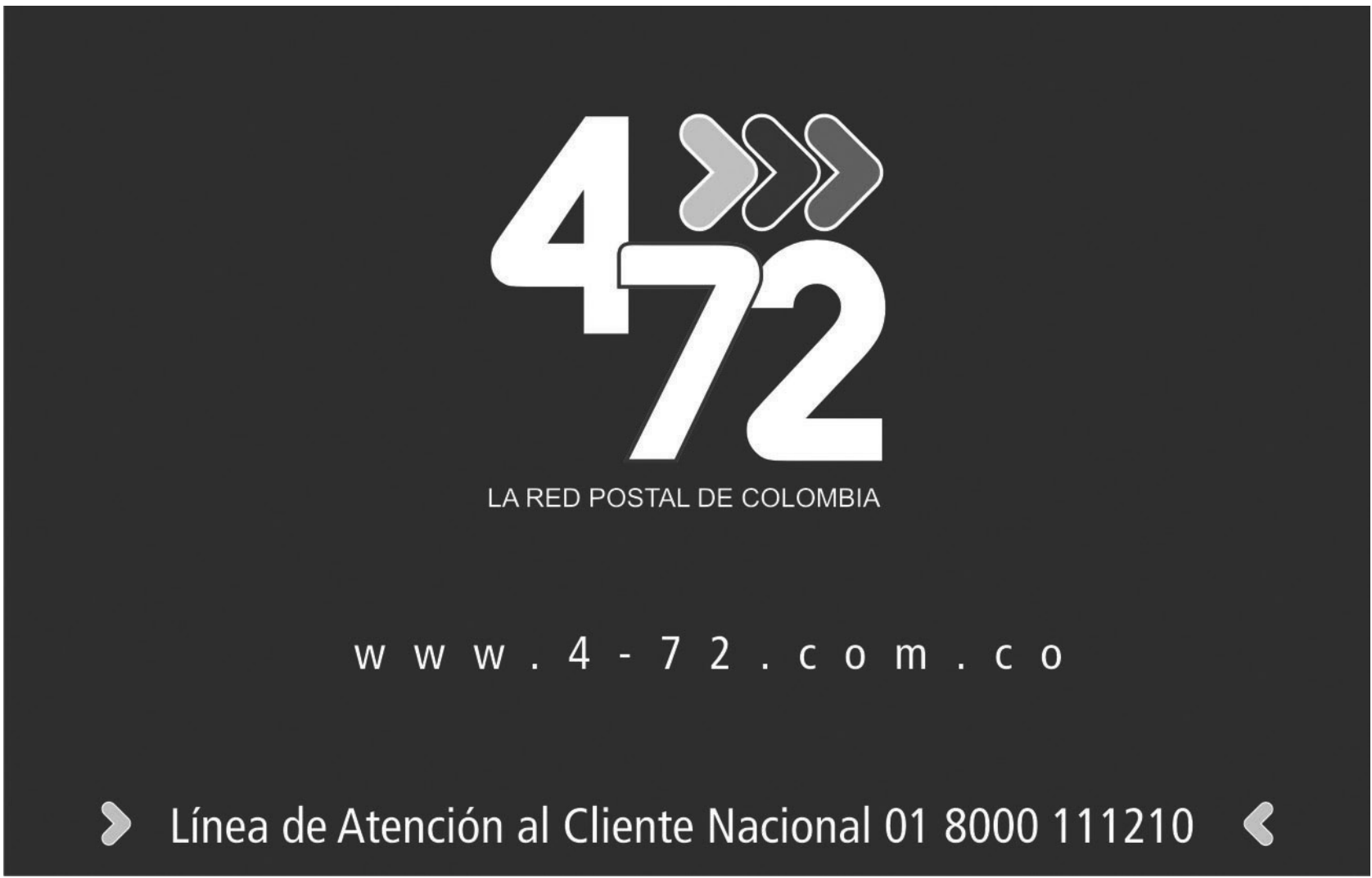




\section{CONCLUSIONES}

L

a existencia de políticas por parte de los gobiernos del mundo, la importancia que en la actualidad tienen las estructuras que son necesarias para proyectos de transferencia, los cambios económicos y sociales globales son algunos de los temas que involucran en la actualidad los proyectos de investigación aplicada, que para algunos autores son concebidos por la necesidades de mercado y/o por las necesidades sociales que vive el mundo actual. Los investigadores, que en su gran mayoría participaron en el estudio, presentan un nivel de escolaridad alto, como el doctorado en diferentes áreas; sus experiencias en investigación en el exterior concedieron contrastar la aplicación de un modelo regional y local que otorgaría la implementación del modelo para llevarlo a la realidad y generar impacto del conocimiento aplicado en la sociedad y el sector empresarial.
Factores como el de la cultura en la investigación evidencian en el diseño una dependencia que permita la sensibilización del investigador universitario con respecto al trabajo colaborativo, a la estructuración de proyectos en integrales y otras variables que arraigan nuestra cultura de trabajo individual, precavido y desconfiado.

Otro factor determinante en el diseño, es la gestión en la investigación. Se encontró que es necesario descentralizar algunos procesos administrativos y crear fundaciones que permitan garantizar efectivamente la transferencia de resultados a través de emprendimiento de mediana tecnología y emprendimiento de alta tecnología.

Esta es una primera aproximación que se somete a la discusión en el desarrollo de un modelo de transferencia de resultados de investigación, basado en la realidad entre la brecha cada vez estrecha por el interés en el tema de los actores: la universidad, la empresa y el Estado. 


\section{REFERENCIAS}

David D. D. (1995). University-industry entrepreneurship: The organization and management of American University Technology Transfer Units. Higher Education, 29(4), 369-384.

Arias, J. y Aristizabal, C. (2011). Transferencia de conocimiento orientada a la innovación social en la relación cienciatecnología y sociedad. Revista Pensamiento y Gestión, 31.

Benavente, J. (2004). Cooperación tecnológica entre universidades y empresas: qué son, cómo operan y cuál es su impacto en Chile. Expansiva, Serie En Foco 21, 1-19.

Calderón, M. (2005). Políticas de transferencia tecnológica para la atracción de recursos en las universidades. Revista de derecho y tecnologías de la información, 3, 1-15.

Correo, J. y Arango, M. (2009). Diseño de una metodología de valoración para proyectos de transferencia tecnológica derivados de grupos de investigación. Caso aplicado a los grupos $I+D+i$ de la Universidad de Antioquia (Trabajo de grado de maestría, Universidad Nacional, Medellín, Colombia).

Etzkowitz, H. y Leydesdorff, L. (2000). The dynamics of innovation: from national systems and "mode 2" to a triple helix of university-industry-government relations. Recuperado de http://users.fmg.uva.nl/lleydesdorff/rp2000/

Gary, P. (2007). ¿Puede la ciencia ser un negocio? Lecciones de la biotecnología. América Latina: Harvard Business Review, pp. 37-43.

García, M. (2012). Estrategias de mercadeo interno para mejorar las condiciones de la problemática organizacional de los institutos de investigación de la Universidad de Pamplona. Revista FACE, VII(11).

García, M. y Duran, J. (2011). El ayer y el hoy de la integración universidad-empresa. Revista FACE, VI(10).

Göktepe, D. (2005). Investigation of university industry technology transfer cases: a conceptual and methodological approach. Working paper, pp. 1-38.

Hernández, C. (2006). Knowledge transfer opportunities for the bioscience sector in Chile.

Jasso, J. (2004). Trayectoria tecnológica y ciclo de vida de las empresas: una interpretación metodológica acerca del rumbo de la innovación. Contaduría y Administración. Recuperado de www.ejournal.unam.mx/rca/rca_index.html

República de Colombia (2009). Ley por la cual se transforma a Colciencias en Departamento Administrativo. Se fortalece el Sistema Nacional de Ciencia, Tecnología e Innovación en Colombia ( $\left.\mathrm{N}^{\mathrm{0}} 1286\right)$. Recuperado de http://www. secretariasenado.gov.co/senado/basedoc/ley/2009/ley_1286_2009.html 
Leydesdorff, L. y Etzkowitz, H. (1998). The Non-linear Dynamics of Sociological Reflections. International Sociology, 25-45.

López, M., Mejía. J. y Schmal, R. (2006). Un acercamiento al concepto de la transferencia de tecnología en las universidades $y$ sus diferentes manifestaciones. Panorama Socioeconómico, 24(32).

Luna, K. y Solleiro, J. L. (2007). La gestión de la propiedad intelectual en centros de investigación mexicanos: el caso del instituto mexicano de petróleo. Journal of technology management \& innovation, 2(2), 157-169.

Morales, S. T. (2008). El emprendedor académico y la decisión de crear spin off: un análisis del caso español (Tesis doctoral, Departamento de dirección de empresas, Universidad de Valencia, Valencia, España, pp. 50).

Ortín, P., Salas, V., Trujillo, M. V. y Vendrell., F. (2008). La creación de spin off universitarias en España: caracteristicas determinantes y resultados. Economía industrial, 368, 79-95.

Solleiro, J. (2008). En búsqueda de un sistema de prácticas para la vinculación exitosa de universidades y centros de $I+D$ con el sector producto. Ponencia presentada en el Foro sobre la relación Universidad-Empresa-Estado en Colombia. Asociación Colombiana de Universidades (ASCUN), Medellín.

Solleiro, J. y Castañón, R. (2008). Gestión tecnológica: conceptos y prácticas. México D.F.; primera edición.

Smith, K. (1995). Les interactions dans les systémes de connaissances: justifications, conséquences au plan de l'action goubernamentale et méthodes emperiques. STI Revue, 16, 75-114.

Rincón de Parra, H. (2004). La evaluación de la transferencia de conocimiento en la relación de cooperación UniversidadEmpresa: una visión desde el contexto de la sociedad de conocimiento. Visión Gerencial, 1(2).

Teruya, D. (2010). El rol de las políticas públicas brasileñas para promover la interacción entre las universidades y las empresas. Seminario sobre políticas públicas para el desarrollo: ley de innovación de Brasil. Colciencias. Programa sistema general de regalías. pp. 70-71.

Tognato, C. (2005). Comercializar la tecnología generada desde las universidades: un reto institucional. Revista de Ingeniería, 21, 30-39.

Vega, J., Fernández, I. y Huanca (2007). ¿La relación Universidad-Empresa en América Latina: apropiación incorrecta de modelos foráneos? Journal of technology management \& innovation, 2(2). 